\title{
Application of advanced light microscopic techniques to gain deeper insights into cheese matrix physico-chemistry
}

\author{
Zuzana Burdikova ${ }^{1} \cdot$ Zdenek Svindrych $^{2}$. \\ Cian Hickey $^{1} \cdot$ Martin G. Wilkinson $^{4}$. \\ Mark A. E. Auty ${ }^{1}$ - Ota Samek ${ }^{3}$ - Silvia Bernatova ${ }^{3}$. \\ Vladislav Krzyzanek ${ }^{3}$ - Ammasi Periasamy ${ }^{2}$. \\ Jeremiah J. Sheehan ${ }^{1}$
}

Received: 9 April 2015 /Revised: 27 July 2015 / Accepted: 28 July 2015 /

Published online: 1 September 2015

(C) INRA and Springer-Verlag France 2015

\begin{abstract}
Despite considerable advances in confocal microscopy and related technologies, advanced light microscopy techniques have been rarely used to study the microstructure of cheese matrices. Here, we demonstrate the potential of advanced microscopic techniques, namely fluorescence lifetime imaging (FLIM), confocal Raman spectroscopy and non-linear microscopy (two-photon excitation and second harmonic generation microscopy), to study localized physicochemical properties of cheese matrices. These methods allow for precise localization of individual chemical components and help to determine their spatial organization in three dimensions. We discuss both fluorescent labelling and label-free methods that provide valuable information about the localized environment. Overall, these new technologies will enable a greater understanding of the influence of manufacturing processes on cheese quality and consistency.
\end{abstract}

Electronic supplementary material The online version of this article (doi:10.1007/s13594-015-0253-2) contains supplementary material, which is available to authorized users.

This paper is part of the Special issue dedicated to the 9th International Cheese Symposium held in Cork, Ireland and organized by Teagasc in collaboration with University College Cork and INRA, 12th \& 13th November 2014.

Zuzana Burdikova

zuzana.burdikova@teagasc.ie

$\triangle$ Jeremiah J. Sheehan

diarmuid.sheehan@teagasc.ie

1 Teagasc Food Research Centre, Moorepark, Fermoy, Co., Cork, Ireland

2 Department of Biology, University of Virginia, Charlottesville, VA, USA

3 VUT, Brno, Czech Republic

4 Department of Life Sciences, University of Limerick, Limerick, Ireland 
Keywords Confocal microscopy $\cdot$ Cheese matrix $\cdot$ Fluorescence lifetime $\cdot$ Second harmonic generation - Two-photon excitation · Confocal Raman microscopy

\section{Introduction}

Cheese is a complex soft matter composed of a protein matrix entrapping fat, water, minerals and dispersed bacterial colonies and their metabolites. The characteristic structure, texture and flavour properties of cheese result from a sequence of dynamic physicochemical, microbial and biochemical processes starting in the cheese vat during manufacture and continuing throughout ripening and cold storage. The textural and rheological properties of a cheese are dependent on interactions between its structural units, including those between fat globules and the protein matrix, fat globules coated with casein micelles which may interact with cheese matrix, moisture content, pools of free fat, levels of proteolysis and casein matrix bond strength (Everett and Auty 2008; Hickey et al. 2015).

Fat may be entrapped in the cheese matrix as inert filler or as a copolymer within the casein matrix. It can exist in globular form, consisting of droplets ranging in size from 0.2 to $15 \mu \mathrm{m}$ with an average of $4 \mu \mathrm{m}$ (Lopez et al. 2007) surrounded by milk fat globular membrane material (MFGM) or it may exist as non-globular free fat. The MFGM is a layer of surface active material which envelops the fat globule and is composed of proteins, enzymes, glycoprotein, triacylglycerols, phospholipids, glycolipids, cholesterol and other minor components (Lopez and Briard-Bion 2007; Hickey et al. 2015). Certain cheese manufacture processes such as high cook temperatures result in coalescence of fat globules along with fracturing of the MFGM during the ensuing pressing of the curds results in the formation of free fat and the release of MFGM material (Lopez and Briard-Bion 2007; Hickey et al. 2015). Amphipolar properties of the phospholipids in the MFGM also enable protein-fat interactions which are essential for matrix structure and enable fat globules to act as emulsifiers within the matrix stabilizing both oil-in-water and water-in-oil emulsions. This is probably responsible for bacterial colonies within the cheese matrix locating at the proteinfat interface in contact with the MFGM due to the hydrophobic nature of LAB bacteria (Banks 1991; Lopez et al. 2006; Pereira et al. 2009; Gallier et al. 2010).

Calcium and phosphorus are the key mineral components within cheese matrices and participate in the protein network that forms through milk coagulation and have a major effect on the structure and texture of cheese (Upreti and Metzger 2006; Lucey and Fox 2015). Increased levels of acidification during cheese manufacture results in conversion of colloidal calcium phosphate to the soluble form which is lost in the whey (Sheehan and Guinee 2004). Excess calcium in the cheese post manufacture can lead to the development of calcium lactate crystals (Upreti and Metzger 2006).

Starter bacteria are integral to cheese manufacture and ripening. Their primary role is to acidify milk during the initial fermentation and then during ripening to lyse thereby releasing protease and lipase enzymes into the cheese matrix resulting in the breakdown of peptides, amino acids and fatty acids leading to the development of volatile flavour compounds (Hickey et al. 2015). The work of Jeanson et al. (2011) showed bacterial colonies to be randomly distributed and static within the cheese matrix, while other studies (Laloy et al. 1996; Lopez et al. 2006) showed bacteria to be located in direct contact with the MFGM or located at the casein-fat interface. During ripening, residual sugars such as lactose and galactose provide substrate for the growth of non-starter lactic acid bacteria (NSLAB), while 
metabolites of starter fermentation such as lactate enable growth of secondary/adjunct cultures such as propionic acid bacteria.

Given the complexity of cheese as a physical and biological system and considering its manufacture from a raw material which can vary markedly in composition, there is a continued need to understand and control the factors that influence cheese quality and consistency. Traditionally, research in the area of cheese microstructure has relied on scanning or transmission electron microscopy and basic confocal microscopy of fluorescently labelled cheese samples. Scanning electron microscopy (SEM) provides detailed topographic images of the cheese surface (or fracture surfaces in the case of freeze-fracture cryo-SEM), differentiating fats, proteins salt crystals and even individual bacterial cells and colonies. However, standard electron microscopy cannot provide complete 3D information about spatial arrangement of individual cheese components. On the other hand, confocal laser scanning microscopy (CLSM), in conjunction with proper fluorescence staining, can provide three-dimensional visualization of fat and protein components or separation of dead and live bacteria inside the cheese matrix (Auty et al. 2001; El-Bakry and Sheehan 2014).

When detailed information about local chemical properties or spatial organization of individual cheese components is required, e.g. individual lipid constituents (triglycerides, phospholipids), distribution of calcium lactate crystals, local $\mathrm{pH}$, or distribution of trace components, advanced microscopic techniques prove themselves to be valuable tools in food research. Thus, with recent development in materials sciences including advanced microscopic techniques, it is now timely to apply these techniques to gain a deeper understanding of cheese matrices and their role in cheese quality and consistency. The objective of this research was to evaluate the application of a range of advanced light microscopic techniques in research into the physicochemical properties of cheese matrices.

\section{Materials and methods}

\subsection{Cheese preparation}

\subsubsection{Starter strains}

Thermophilic starter cultures typically used in Swiss-type cheese manufacture (Scott 1981), i.e. S. thermophilus TH3 (ST) and L. helveticus LHB02 (LH), were purchased from Chr. Hansen Ltd. (Little Island, Co. Cork, Ireland) as individual frozen concentrates and stored at $-80{ }^{\circ} \mathrm{C}$ until cheese manufacture.

\subsubsection{Cheese manufacture}

Raw milk was obtained from a local dairy company, standardized to a protein to fat ratio of 1.01:1, held overnight at $<10{ }^{\circ} \mathrm{C}$, pasteurized at $72{ }^{\circ} \mathrm{C}$ for $15 \mathrm{~s}$, and pumped at $32{ }^{\circ} \mathrm{C}$ into cylindrical jacketed stainless steel vat $(500 \mathrm{~L})$ with automated variable speed cutting and stirring equipment (APV, Schweiz AG, Worb, Switzerland). Cheesemilk was heated to $34{ }^{\circ} \mathrm{C}$ and inoculated with $10 \mathrm{mg} . \mathrm{L}^{-1}$ of frozen direct vat starter (DVS) ST and $5 \mathrm{mg} . \mathrm{L}^{-1}$ of frozen DVS LH (Chr. Hansens Ltd.). After a 60-min ripening period, chymosin (Chymax plus, Chr. Hansens Ltd.) diluted to 1:6 with de-ionized water, was added at a level of $18 \mathrm{~mL}$ per $100 \mathrm{~kg}$ of milk. A coagulation time of 35 min was allowed prior to cutting of the 
coagulum. After a 10-min healing period, the curd/whey mixture was cooked by steam injection into the jacket of the vat with constant stirring. Maximum scald employed was $50{ }^{\circ} \mathrm{C}$. Curds were cooked at a rate of $0.5^{\circ} \mathrm{C}$.min from 34 to $45^{\circ} \mathrm{C}$ and at $1{ }^{\circ} \mathrm{C}$.min from $45^{\circ} \mathrm{C}$ to maximum scald. Curds were pitched at $\mathrm{pH}$ 6.3.

The curds were drained, cheddared, milled at $\mathrm{pH} 5.3$, salted at a rate of $1.45 \%(w / w)$ and pressed overnight on a horizontal press at $264.6 \mathrm{kPa}$ in $20 \mathrm{~kg}$ blocks. The cheeses were then vacuum packed and ripened at $8{ }^{\circ} \mathrm{C}$ for up to 230 days.

\subsubsection{Cheese preparation for light microscopy}

Thin slices of cheese, measuring approximately $5 \times 5 \times 3 \mathrm{~mm}$, were freshly cut from a cheese sample using a scalpel.

Neutral lipids were stained with a lipid specific Nile blue fluorescent dye (SigmaAldrich, St. Louis, MO), prepared in acetone with concentration of $42 \mu \mathrm{g} \cdot \mathrm{mL}^{-1}$.

Phospholipids were labelled with N-(lyssamine Rhodamine-B sulfonyl)-1,2dioleoyl-sn-glycero-3-phosphoethanolamine (Rh-DOPE; Avanti Polar Lipids Inc., Birmingham, England), dispersed in chloroform. To stain phospholipids, about $200 \mu \mathrm{L}$ of the staining of Rh-DOPE was added to the coverslip. After chloroform evaporation, the coverslip was pressed against the slice of cheese to permit diffusion of the stain for $30 \mathrm{~min}$ in the dark at $4{ }^{\circ} \mathrm{C}$.

\subsection{Advanced techniques in optical microscopy: basic principles and instrumentation}

\subsubsection{Confocal laser scanning fluorescence microscopy}

Confocal laser scanning fluorescence microscopy (CLSM) has long been a standard tool in cell biology and became popular in other areas of science, including food research. It is based on fluorescence, a phenomenon where a photon of suitable wavelength is absorbed by a specific dye molecule, followed by an emission of a photon with somewhat longer wavelength (lower energy). With a set of fluorescence filters, the distribution of the fluorescent can be observed with high contrast and specificity. The main disadvantage of simple widefield fluorescence observation is the blur and haze caused by out-of-focus fluorescence, particularly in thick samples, a common situation in food research. The elaborate optical arrangement of a CLSM effectively blocks the out-of-focus light, employing laser excitation of a single spot in the sample, fast raster scanning in three dimensions and efficient multichannel detectors (Pawley 2006). With a CLSM, one obtains a full 3D representation of the spatial distribution of fluorescent molecules in the sample with high contrast and resolution.

The CLSM is a basic platform that can be easily extended to perform advanced microscopic imaging, such as two-photon excitation, second harmonic generation, fluorescence lifetime or Raman microscopy.

\subsubsection{Fluorescence lifetime imaging microscopy}

Fluorescence lifetime imaging microscopy (FLIM) measures the characteristic time a fluorescent molecule spends in the excited state (fluorescence lifetime). This lifetime is 
typically a few nanoseconds in length and it is specific for given fluorophore. Moreover, the fluorescence lifetime of many fluorescent dyes is a sensitive indicator of local chemical properties, such as ion concentration, $\mathrm{pH}$, oxygen concentration or presence of other fluorescent molecules (Lakowicz 2006). Consequently, FLIM can not only be used to distinguish different fluorophores on the basis of their characteristic lifetimes (rather than their spectral properties) but also to distinguish among different environments based on changes in lifetime of the same fluorophore, for example local $\mathrm{pH}$ within the cheese matrix (Burdikova et al. 2015). Fluorescence lifetime microscopy can also provide additional valuable information in fat composition studies when combined with various fluorescent dyes (e.g. Nile blue, Rhodamine-DOPE). Better understanding of the composition of lipid droplets in cheese matrix may improve the functional and nutritional properties of food products (Lopez and Briard-Bion 2007).

Fluorescence lifetime measurements of Nile blue- and Rhodamine-DOPE-stained cheese samples were performed with Zeiss LSM780 NLO confocal microscope based on Axio Observer. Z1 inverted microscope (Carl Zeiss MicroImaging, GmbH) equipped with Chameleon Vision (Coherent, Inc.) pulsed IR laser (0.1 ps pulses, $80 \mathrm{MHz}$ repetition rate) tuned to $860 \mathrm{~nm}$ for two-photon fluorescence excitation and Becker \& Hickl hybrid detector (HPM-100-40) and TCSPC hardware (SPC-150, Becker \& Hickl, GmbH). Fluorescence was collected through an IR-blocking filter and 580-640 nm emission filter with a Plan Apo 63×/1.4 oil IR objective lens. The measured fluorescence decay curves were analyzed with Becker \& Hickl SPCImage fitting software to yield the mean fluorescence lifetime in $256 \times 256$ pixel lifetime images.

\subsubsection{Two-photon excitation and second harmonic generation imaging microscopy}

Second harmonic generation (SHG) imaging is non-linear optical process with two requirements - high excitation intensity and non-centrosymmetric molecules with long range order present in the sample (Bianchini and Diaspro 2008). No fluorescence takes place in this process and the wavelength of the emitted light (as the name suggests) is exactly one half of the excitation wavelength. Generally, the same pulsed laser source used for two-photon excitation (TPE) is also suitable for SHG, and these two signals can be easily separated spectrally. As no labelling is needed for SHG imaging (Diaspro et al. 2002), it is a vital method for visualizing certain specific components of the sample, e.g. collagen in tissues (Filová et al. 2010; Šepitka et al. 2012). Some low symmetry crystals also emit strong SHG signal (Haupert et al. 2012) so this technique can be applied to detect calcium lactate crystals within cheese matrices.

TPE is a non-linear process where a fluorescent molecule simultaneously absorbs two photons of low energy (long wavelength, typically in the near infrared part of the spectrum) in order to reach an exited state. The dye molecule then emits a photon, whose energy is somewhat lower than the sum of the excitation photon energies (the emission wavelength is longer than one half of the excitation wavelength). However, for this phenomenon to appear, the excitation intensity must be exceptionally high (megawatts per square micrometre) and this is only achievable with dedicated pulsed lasers (pulses of the order of picoseconds or shorter) in a laser point scanning setup (Diaspro et al. 2006). The main advantages of two-photon excitation microscopy are (i) lower absorption and scattering of the IR excitation light in many kinds of samples (e.g. 
biological tissues), (ii) due to non-linear effects, no fluorescence originates from outside the focus plane (Burdíková et al. 2010), so there is no need to block the out-of-focus light, and this greatly enhances the fluorescence collection efficiency in highly scattering samples. These two effects together facilitate fluorescence microscopy deep within a scattering sample.

SHG signal of calcium lactate crystals, together with TPE fluorescence, were collected with TCS SP8 AOBS MP confocal microscope (Leica Microsystems, GmbH) equipped with InSight DeepSee (Spectra-Physics, Inc.) pulsed IR laser ( 0.1 ps pulses, $80 \mathrm{MHz}$ repetition rate) tuned to $860 \mathrm{~nm}$. Internal spectral detectors were used for fluorescence acquisition and the frequency doubled signal was observed through 420 $440 \mathrm{~nm}$ bandpass filter in transmission geometry.

\subsubsection{Raman confocal imaging}

Raman scattering is a process of inelastic scattering of photons, where part of the photon energy is transferred to molecular vibrations. It is a very weak process (typically less than $1 \mathrm{ppm}$ of incoming photons are inelastically scattered), however, the spectrum of the scattered photons (Raman spectrum) shows a very high level of detail. Every molecule in the sample has its characteristic spectrum, reflecting the energies of vibrational modes of that molecule. With known spectra of individual constituents (often found in specialized databases), one can decompose the measured Raman spectrum (using, e.g. principal component analysis (PCA) (Shinzawa et al. 2009)) into the contributions of the individual chemical species. When combined with a confocal microscopic setup, the method (Hollricher and Ibach 2011) is a powerful tool to determine the chemical composition of the sample with micrometre resolution, in 3D and without any labelling. Here, we show the potential and limitations of this technique in determining the local composition of cheese samples.

A slice of cheese was cut using a sharp knife and the local Raman spectrum was taken using inVia confocal Raman microscope (Renishaw, PLC) with $785 \mathrm{~nm}$ excitation (laser power of about $60 \mathrm{~mW}$ in the sample plane) and 20× objective. Laser spot is visible in the middle of Fig. 5. Vertical size of the laser spot is about $20 \mu \mathrm{m}$.

In order to extract quantitative information from the acquired Raman spectra, the statistical classification of the spectra was carried out adopting a rolling-circle procedure for spectral processing. In this way, the slowly varying spectral background is suppressed. Also automated subtraction of cosmic ray peaks, and the Savitzky-Golay procedure for subsequent noise filtering was employed. Finally, all the data obtained from the studied samples was analyzed using standard principal component analysis (PCA) algorithms in order to distinguish possible difference between them.

\subsubsection{Cryo-electron microscopy}

Cheese samples were imaged in a scanning electron microscope using an Alto2500 cryo-stage system (Gatan UK Ltd., Abingdon, UK.). Sections of cheese $1 \times 1 \times 4 \mathrm{~mm}$ were cut at room temperature with a razor blade, then plunge frozen in liquid nitrogen slush at $-207{ }^{\circ} \mathrm{C}$, followed by freeze fracturing under vacuum in the cryo-chamber using a precooled scalpel blade. The fractured surfaces were then etched at $-85^{\circ} \mathrm{C}$ for $15 \mathrm{~min}$, cooled to $-125^{\circ} \mathrm{C}$, and coated with sputtered platinum $(10 \mathrm{~mA}$ for $60 \mathrm{~s})$. 
Samples were transferred under vacuum to the cold stage in a Zeiss Supra 40VP field emission scanning electron microscope (Carl Zeiss AG, Darmstadt, Germany), where they were maintained at $-125^{\circ} \mathrm{C}$ and imaged using an accelerating voltage of $1 \mathrm{kV}$.

\section{Results and discussion}

\subsection{Calcium lactate crystals visualized with SHG microscopy}

To our knowledge, it is the first time the SGH microscopy has been applied to examine dairy matrices of any product type and in particular to visualize calcium lactate crystals placed inside cheese matrix without labelling. In Fig. 1, we compare SHG images of the crystals, overlaid with TPE fluorescence of the cheese matrix (Fig. 1a, see also the animation in Online Resource 1) with the already established methodology in cheese research-cryo-SEM (Fig. 1b). The lower resolution of SHG is clearly offset by straightforward sample preparation, the depth resolution and the possibility to combine SHG with other fluorescence staining methods (e.g. Nile blue) and to study the mutual spatial organization of the individual cheese components.

The SHG signal also depends on the polarization of excitation light with respect to crystallographic orientation of the crystals. To demonstrate this, we rotated the polarization of the excitation light and acquired the SHG images at angles $0^{\circ}$ (green), $30^{\circ}$ (red) and $60^{\circ}$ (blue), Fig. 2a, b. The merge of all tree angles provides complex information about the lactate calcium crystal location, shape and orientation with particular reference to the protein and fat components of the cheese matrix. Thus, it provides a greater visual understanding of the relationship between calcium lactate crystals and other constituents of the cheese matrix.

Calcium lactate crystals form when $\mathrm{Ca}^{2+}$ and lactate ions exceed their solubility, supersaturate the serum phase of the cheese and then crystallize at nucleation sites within cheese matrices. Most starter bacteria ferment lactose to L-lactate, however, considerable amounts of D-lactate are formed by the activity of NSLAB during cheese ripening. The lower solubility of Ca-D-lactate then causes crystal formation (Lawrence et al. 2004). Crystallization of calcium lactate on the surface of Cheddar cheese is a
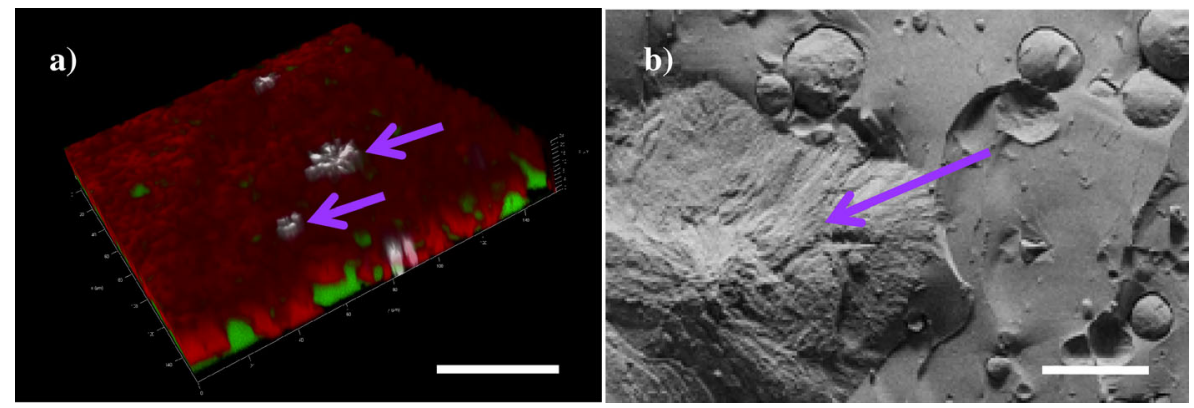

Fig. 1 Calcium lactate crystals inside the Cheddar cheese matrix. a (red) two-photon fluorescence of protein matrix stained with Nile blue; (green) two-photon fluorescence of fat stained with Nile blue; (grey) SHG signal from the calcium lactate crystals (purple arrows); scale bar $50 \mu \mathrm{m}$. b Freeze-fracture cryo-SEM image showing the details of fat droplets in protein matrix and a single calcium lactate crystal (purple arrow); scale bar $5 \mu \mathrm{m}$

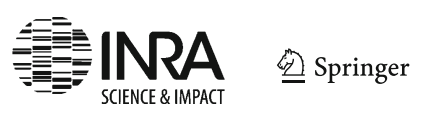




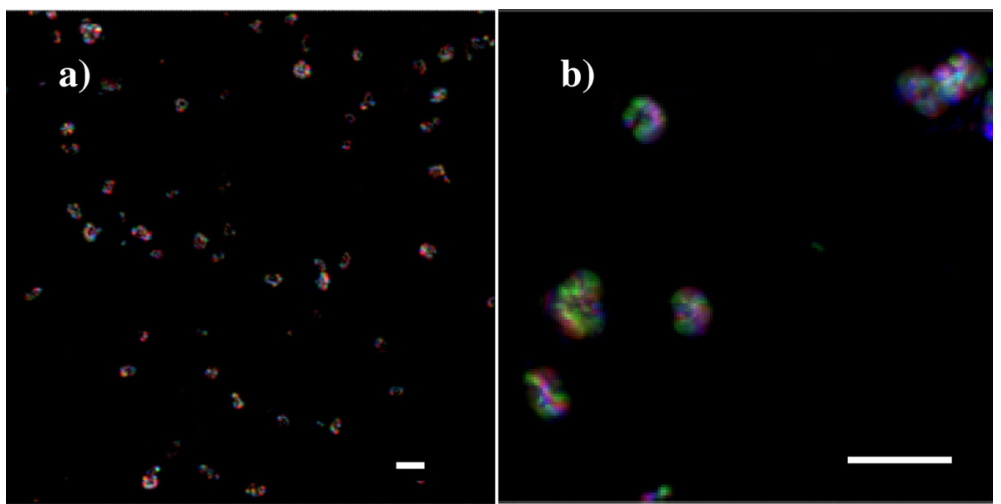

Fig. 2 a Calcium lactate crystals inside the Cheddar cheese matrix visualized using SHG microscopy technique. The polarization angles of the incident light were color coded as green $\left(0^{\circ}\right)$, red $\left(30^{\circ}\right)$ and blue $\left(60^{\circ}\right)$. b Detail of individual crystals. Scale bars $20 \mu \mathrm{m}$

common and troublesome defect (Pearce et al. 1973). The deposits are visible to the naked eye; products containing crystal deposits are not aesthetically pleasing and can be sometimes rejected by the retailer/consumer who may confuse them with mould contamination.

Calcium lactate crystals can be visualized using X-ray crystallography but we will miss the chemical information about the placement fat and protein around the crystals. The crystalline deposits are also frequently observed by standard light microscopy SEM or TEM in processed cheese products (Kalab 1995). Using various techniques such as electron microscopy and X-Ray diffraction analysis, infrared spectroscopy and energy dispersive X-Ray spectrometry analysis (EDSA), several crystal types have been identified, e.g. monoclinic calcium pyrophosphate dehydrate, disodium phosphate dodecahydrate, unreacted melting salts, tyrosine, calcium citrate, fatty acids, protein and lactose (Kondo et al. 1990). The composition of crystalline structures in natural cheeses and PCPs was studied by with SEM EDSA and X-Ray diffraction (Washam et al. 1985). Calcium specific fluorescent stain Alizarin Red was used to identify calcium lactate crystals by optical microscopy (Yiu 1985).

In comparison, SHG microscopy does not allow us to chemically characterize the composition of crystals, but this label-free microscopic technique allows us to visualize the calcium lactate crystals inside cheese matrix, and with combination with standard CLSM, we can get also the information about the relationship between crystals and the surrounding environment like location of fat, protein, phospholipids, live/dead bacteria in three dimensions (Online Resource 1). This localization after further investigation can further illustrate the relationship between calcium lactate crystals and bacterial, fat and mineral placement inside the cheese matrix.

\subsection{Lipids visualized with FLIM}

It is strongly evident that the sensitivity of the spectral properties of Nile blue fluorescent dye towards the polarity of the local environment (Jose and Burgess 2006), as shown in Fig. 3a, b, enables the analyst to determine with much greater clarity and precision the dispersion and morphology of fat within the cheese matrix. As 


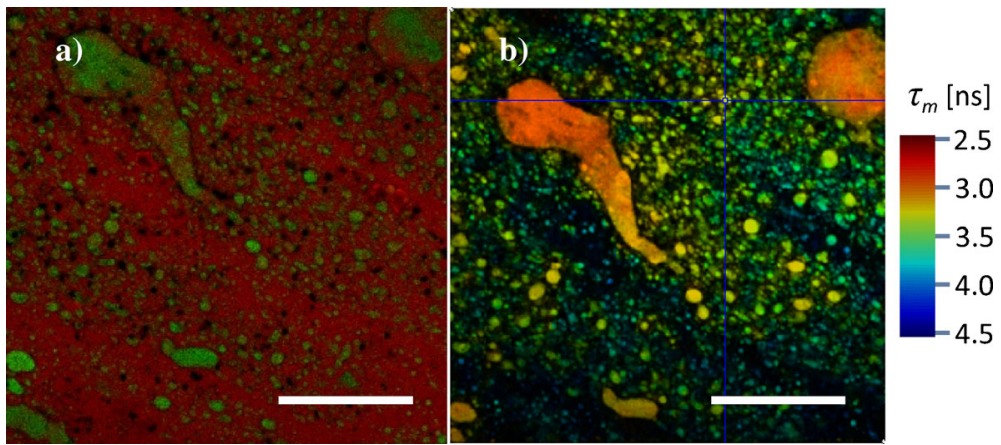

Fig. 3 a Confocal image of the Cheddar cheese surface; Nile blue staining: (green) fat, (red) protein matrix. b Fluorescence lifetime of the Nile blue-stained cheese surface in the 520-570-nm emission band, corresponding to non-polar (fat) environment. The pseudocolor encodes the local fluorescence lifetime. Scale bars $20 \mu \mathrm{m}$

expected, fat in the cheese matrix illustrated in Fig. 3 is comprised of both fat in globular form and non-globular free fat. It may also be considered that the results of our FLIM studies of the lipid phase show strong correlation between the fluorescence lifetime and the size of the individual lipid droplets (Fig. 3b). This phenomenon deserves more investigation, as it may suggest that the composition of lipid droplets may depend on their size, or vice versa.

Based on the work of Lopez et al. (2008), we also applied the fluorescent marker Rhodamine-DOPE for phospholipids, i.e. lipids that can form lipid bilayers due to the presence of hydrophilic head, in addition to their hydrophobic tail. Figure $4 \mathrm{a}, \mathrm{b}$ acquired by CLSM show the occurrence of phospholipids on the edge between the fat droplets and the protein matrix. The corresponding FLIM image shows additional details of phospholipids placement (Fig. 4c), additional visual information is conveyed by the pseudocolor, encoding the local fluorescence lifetime.

Currently it is possible to detect phospholipids using spectroscopy, nuclear magnetic resonance spectroscopy, particularly with P-NMR. With HPLC-ELSD, it is possible to determine the composition and type of phospholipids present, but it does not provide information about the placement and location of the phospholipids in within the cheese matrix. Utilization of FLIM methodology provides more accurate information about the placement of phospholipids in the cheese matrix. To achieve separation of different types of fats inside the cheese matrix, we used the same methods as used previously (Lopez et al. 2008) for CLSM.
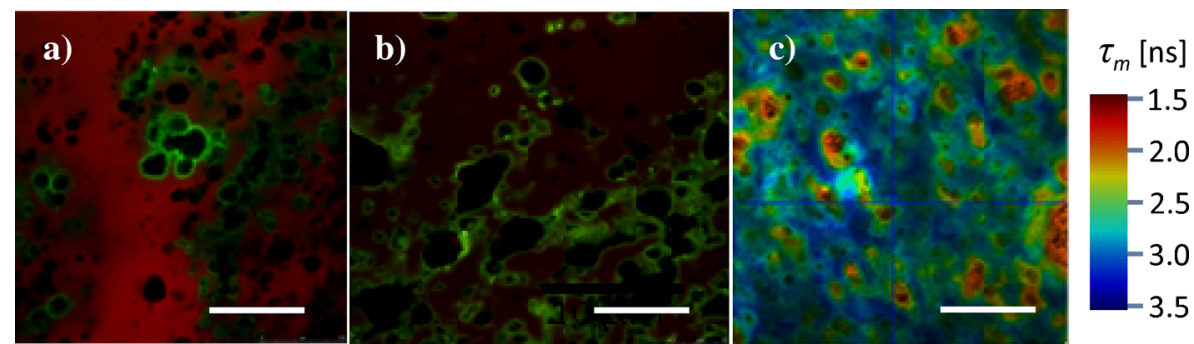

Fig. 4 a, b Phospholipids visualized by Rhodamine-DOPE (green) and protein matrix (red), Cheddar cheese, CLSM; c FLIM image of the phospholipids (Rhodamine-DOPE). The pseudocolor encodes the local fluorescence lifetime. Scale bars $20 \mu \mathrm{m}$ 


\subsection{Characterization of chemical composition of cheese matrix by confocal Raman microscopy}

This method offers the capability to determine variability in chemical compositions arising during cheese manufacture, which in the long term, offers the potential for developing rapid or real-time in situ process analysis and control methodologies. Moreover, we have demonstrated that it is possible to reveal the contribution of fatty acids and proteins (Figs. 5 and 6) to the overall statistical variance within the chemical fingerprint using PCA so that different types of cheese can be distinguished. The results suggest that Raman spectroscopy analysis has the potential for the characterization of cheese without any time-consuming sample preparation (see sample preparation for experiments at Fig. 5). This could be of benefit in the food industry where Raman spectroscopy can be used as a quality sensor of selected products directly at the production line rapidly sensing any changes in chemical composition.

To demonstrate the capability of Raman spectroscopy for the compositional analysis and the spatial visualization of cheese samples, we have recently performed experiments in which spectra were acquired point-by-point, at a few selected positions on a cut sample surface (Fig. 5). Consequently, some relevant molecular complexes (Fig. 6) could be identified in this way, with some spatial resolution so that e.g. inhomogeneous distribution of beta-carotenoids is visible. As an example, this analytical approach could help to determine the underlying causes of the development of a pink color defect, persistent in a wide range of ripened cheese varieties (Daly et al. 2012).

Raman spectroscopy allows the clear identification of particular biological molecular compounds within a complex spectrum which are responsible for changes in the
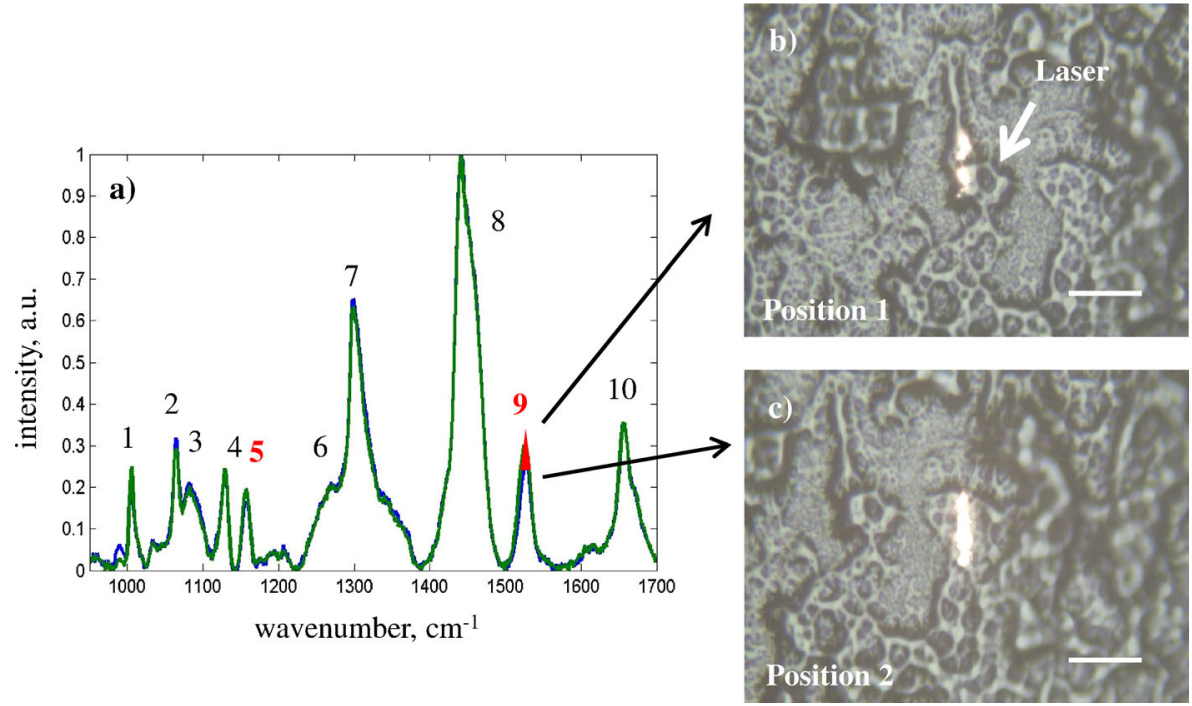

Fig. 5 a Raman spectra of Cheddar cheese taken from two different positions of a cut cheese surface; b, c positions from which the spectra were taken - in the middle of each position, a laser trace is shown (this is the exact location from which data were taken). Note the arrows showing differences in beta-carotene peaks - indicating its inhomogeneous distribution. The vertical size of the laser spot is about $20 \mu \mathrm{m}$. Samples for analysis were achieved by preparing very thin slices and placing directly under a microscope where Raman spectrum was taken. Scale bars $20 \mu \mathrm{m}$ 


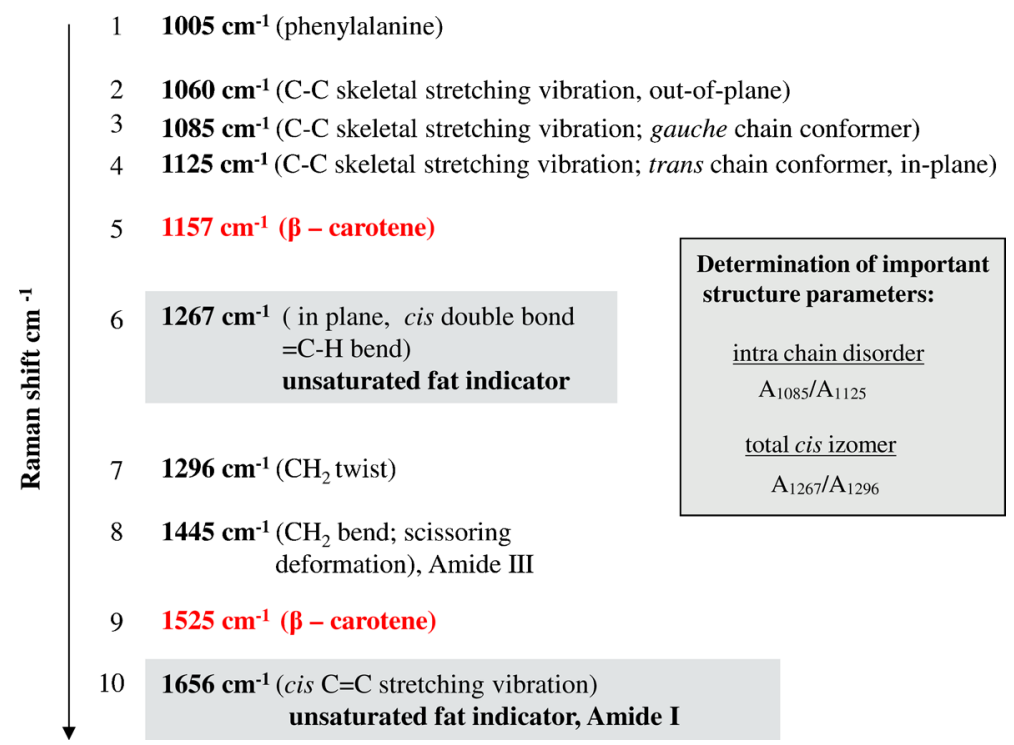

Fig. 6 Summary of prominent peaks observed in the Raman spectra of Cheddar cheese, together with suggested assignment of chemical compounds. The peak numbers of the table are used to identify features in the spectra shown in Fig. 5. Determination of selected structure parameters is highlighted in the inset

matrix (for example, in cheese matrices fats and protein). Raman spectroscopy can be regarded as the method of choice for analysis of biological/food samples (Maquelin et al. 2002; Harz et al. 2005; Bernatová et al. 2013; Samek et al. 2014). Two previous reviews provide valuable information on Raman spectroscopy studies of the molecular complexes in biological specimen (Movasaghi et al. 2007; Notingher 2007). In

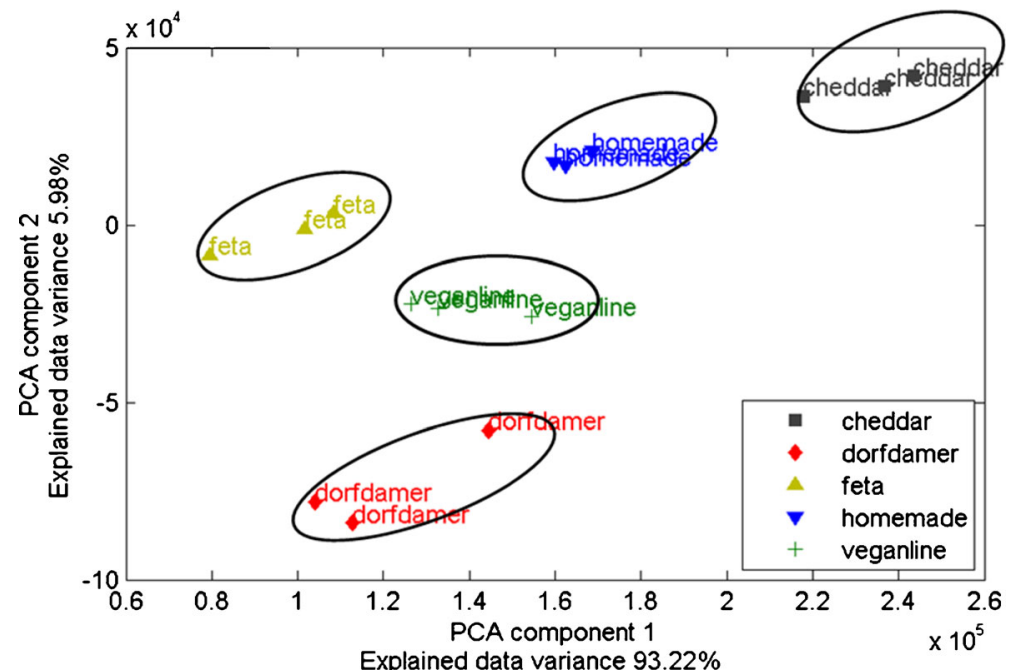

Fig. 7 Scores plot of the first two principal components for five different types of cheese. With PCA, one can clearly separate the clusters of spectra associated with the different types of cheese. Cheese types were selected/bought in the local shop as follows: Cheddar, home-made cheese from a local farmer, Dorfdamer, Feta and Veganline 
addition, a reasonably detailed database of Raman features encountered in biological samples was published (De Gelder et al. 2007). Subsequent spectral decomposition or blind principal component analysis (PCA) can stand as a basis for discerning important features of the studied samples, as exemplified in Fig. 7.

However, this type of localized investigation for species and/or compound identification is time consuming and often does not collect sufficient or complete (multispectra) information about the whole sample, particularly where there is likely to by inhomogeneity within the sample. A possible solution to the problem of localized measurements, to build up a compositional map, has come about with the commercial introduction of line-scan techniques. This approach is around for some time, and has recently been applied to the study of biological samples (Samek et al. 2010). In essence, rather than measuring the sample point-by-point the technique relies on line scanning in which multiple locations are sampled simultaneously. With the many, rapidly acquired spectra - up to tens of thousands of spectra — 'data mining' for analysis and identification becomes possible, using standard chemometric techniques. This can be also combined with confocal imaging to quickly build up 3D maps of the surface.

\section{Conclusion}

Given the desire within the cheese industry to achieve a greater understanding of the influence of manufacture and ripening parameters on cheese quality and consistency, there is a continued interest in exploiting new analytical techniques to gain new insights into the physicochemical characteristics of cheese matrices. In this study, a range of advanced light microscopic techniques were evaluated to determine their potential to provide a better understanding of cheese matrices at the micro-scale and in particular to aid research into determining the level of localized inhomogeneity within the cheese matrix. This study concluded that (i) second harmonic generation microscopy technique facilitates visualization of calcium lactate crystals with reference to the fat and protein components within the cheese matrix; (ii) two-photon excitation fluorescent lifetime microscopic techniques enables a more precise determination and location of fats and phospholipids within the cheese matrix, and (iii) the Raman confocal microscopy offers a new and very powerful methodology to analyze and fingerprint different chemical components of the cheese matrix.

Acknowledgments This work was funded by the Dairy Levy Trust, Ireland, with additional support from the Ministry of Education, Youth and Sports of the Czech Republic (LO1212) together with the European Commission (ALISI No. CZ.1.05/2.1.00/01.0017). We also thank Dr. Jana Pala (Leica Microsystem, Mannhein, Germany) for image acquisition on Leica TCS SP8 microscope and the W. M. Keck Center for Cellular Imaging (KCCI) at the University of Virginia for expert advice regarding fluorescence lifetime imaging.

Conflict of interest Zuzana Burdikova, Zdenek Svindrych, Cian Hickey, Martin G. Wilkinson, Mark A. E. Auty, Ota Samek, Silvia Bernatova, Vladislav Krzyzanek, Ammasi Periasamy, and Jeremiah J. Sheehan declare that they have no conflict of interest.

Statement of human and animal rights

This article does not contain any studies with human or animal subjects performed by any of the authors. 


\section{References}

Auty MAE, Gardiner GE, McBrearty SJ et al (2001) Direct in situ viability assessment of bacteria in probiotic dairy products using viability staining in conjunction with confocal scanning laser microscopy. Appl Environ Microbiol 67:420-425. doi:10.1128/AEM.67.1.420-425.2001

Banks W (1991) Milk lipids. Bull Int Dairy Fed 260:3-6

Bernatová S, Samek O, Pilát Z et al (2013) Following the mechanisms of bacteriostatic versus bactericidal action using Raman spectroscopy. Molecules 18:13188-13199. doi:10.3390/molecules181113188

Bianchini P, Diaspro A (2008) Three-dimensional (3D) backward and forward second harmonic generation (SHG) microscopy of biological tissues. J Biophotonics 1:443-450. doi:10.1002/jbio.200810060

Burdíková Z, Čapek M, Ostašov P et al (2010) Testate amoebae examined by confocal and two-photon microscopy: implications for taxonomy and ecophysiology. Microsc Microanal 16:735-746

Burdikova Z, Svindrych Z, Pala J et al (2015) Measurement of pH micro-heterogeneity in natural cheese matrices by fluorescence lifetime imaging. Front Microbiol. doi:10.3389/fmicb.2015.00183

Daly DFM, McSweeney PLH, Sheehan JJ (2012) Pink discolouration defect in commercial cheese: a review. Dairy Sci Technol 92:439-453. doi:10.1007/s13594-012-0079-0

De Gelder J, De Gussem K, Vandenabeele P, Moens L (2007) Reference database of Raman spectra of biological molecules. J Raman Spectrosc 38:1133-1147. doi:10.1002/jrs.1734

Diaspro A, Fronte P, Raimondo M, et al. (2002) Functional imaging of living Paramecium by means of confocal and two-photon excitation fluorescence microscopy. pp 24-31

Diaspro A, Bianchini P, Vicidomini G et al (2006) Multi-photon excitation microscopy. Biomed Eng Online 5: 36. doi:10.1186/1475-925X-5-36

El-Bakry M, Sheehan J (2014) Analysing cheese microstructure: a review of recent developments. J Food Eng 125:84-96. doi:10.1016/j.jfoodeng.2013.10.030

Everett DW, Auty MAE (2008) Cheese structure and current methods of analysis. Int Dairy J 18:759-773. doi: 10.1016/j.idairyj.2008.03.012

Filová E, Burdíková Z, Rampichová M et al (2010) Analysis and three-dimensional visualization of collagen in artificial scaffolds using nonlinear microscopy techniques. J Biomed Opt 15:66011-66017

Gallier S, Gragson D, Jiménez-Flores R, Everett D (2010) Using confocal laser scanning microscopy to probe the milk fat globule membrane and associated proteins. J Agric Food Chem 58:4250-4257. doi:10.1021/ jf9032409

Harz M, Rosch P, Peschke K-D et al (2005) Micro-Raman spectroscopic identification of bacterial cells of the genus Staphylococcus and dependence on their cultivation conditions. Analyst 130:1543-1550. doi:10. 1039/B507715J

Haupert LM, DeWalt EL, Simpson GJ (2012) Modeling the SHG activities of diverse protein crystals. Acta Crystallogr Sect D: Biol Crystallogr 68:1513-1521. doi:10.1107/S0907444912037638

Hickey CD, Sheehan JJ, Wilkinson MG, Auty MAE (2015) Growth and location of bacterial colonies within dairy foods using microscopy techniques: a review. Front Microbiol. doi:10.3389/fmicb.2015.00099

Hollricher O, Ibach W (2011) Confocal Raman microscopy. Springer, Berlin

Jeanson S, Chadœuf J, Madec MN et al (2011) Spatial distribution of bacterial colonies in a model cheese. Appl Environ Microbiol 77:1493-500. doi:10.1128/AEM.02233-10

Jose J, Burgess K (2006) Benzophenoxazine-based fluorescent dyes for labeling biomolecules. Tetrahedron 62:11021-11037. doi:10.1016/j.tet.2006.08.056

Kalab M (1995) Practical aspects of electron microscopy in cheese research. In: Malin EL, Tunick MH (eds) Chemistry of structure-function relationships in cheese. Plenum Press, New York, pp 247-276

Kondo Y, Watanabe T, Aita S (1990) Application of the frozen thin sectioning method to the observation and $\mathrm{X}$-ray microanalysis of cheese using an analytical transmission electron microscope. J Jpn Soc Food Sci Technol 37:45-47

Lakowicz JR (2006) Principles of fluorescence spectroscopy, 3rd edn

Laloy E, Vuillemard JC, El Soda ME, Simard RE (1996) Influence of the fat content of Cheddar cheese on retention and localization of starters. Int Dairy J 6:729-740. doi:10.1016/0958-6946(95)00068-2

Lawrence RC, Gilles J, Creamer LK et al (2004) Cheddar cheese and related dry-salted cheese varieties, 3rd edn. Elsevier Academic Press, London

Lopez C, Briard-Bion V (2007) The composition, supramolecular organisation and thermal properties of milk fat: a new challenge for the quality of food products. Lait 87:317-336

Lopez C, Maillard MB, Briard-Bion V et al (2006) Lipolysis during ripening of Emmental cheese considering organization of fat and preferential localization of bacteria. J Agric Food Chem 54:5855-5867. doi:10. 1021/jf0602141

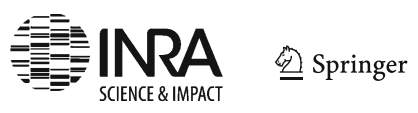


Lopez C, Camier B, Gassi J-Y (2007) Development of the milk fat microstructure during the manufacture and ripening of Emmental cheese observed by confocal laser scanning microscopy. Int Dairy J 17:235-247. doi:10.1016/j.idairyj.2005.12.015

Lopez C, Briard-Bion V, Beaucher E, Ollivon M (2008) Multiscale characterization of the organization of triglycerides and phospholipids in Emmental cheese: from the microscopic to the molecular level. J Agric Food Chem 56:2406-2414. doi:10.1021/jf0720382

Lucey JA, Fox PF (2015) Importance of calcium and phosphate in cheese manufacture: a review. J Dairy Sci 76:1714-1724. doi:10.3168/jds.S0022-0302(93)77504-9

Maquelin K, Kirschner C, Choo-Smith LP et al (2002) Identification of medically relevant microorganisms by vibrational spectroscopy. J Microbiol Methods 51:255-271

Movasaghi Z, Rehman S, Rehman IU (2007) Raman spectroscopy of biological tissues. Appl Spectrosc Rev 42:493-541

Notingher I (2007) Raman spectroscopy cell-based biosensors. Sensors 7:1343-1358

Pawley JB (2006) Handbook of biological confocal microscopy, 3rd edn. Springer Science \& Business Media, New York

Pearce KN, Creamer LK, Gilles J (1973) Calcium lactate deposits on rindless cheddar cheese. N Z J Dairy Sci Technol 8:3-7

Pereira CI, Gomes AMP, Xavier Malcata F (2009) Microstructure of cheese: processing, technological and microbiological considerations. Trends Food Sci Technol 20:213-219. doi:10.1016/j.tifs.2009.02.006

Samek O, Al-Marashi JFM, Telle HH (2010) The potential of Raman spectroscopy for the identification of biofilm formation by Staphylococcus epidermidis. Laser Phys Lett 7:378-383. doi:10.1002/lapl. 200910154

Samek O, Mlynariková K, Bernatová S et al (2014) Candida parapsilosis biofilm identification by Raman spectroscopy. Int J Mol Sci 15:23924-23935. doi:10.3390/ijms151223924

Scott R (1981) Cheesemaking practice. Elsevier Applied Science Publishers, London

Šepitka J, Lukeš J, Staněk L et al (2012) Nanoindentation of intervertebral disc tissues localised by SHG imaging. Comput Methods Biomech Biomed Eng 15:335-336. doi:10.1080/10255842.2012.713601

Sheehan JJ, Guinee TP (2004) Effect of $\mathrm{pH}$ and calcium level on the biochemical, textural and functional properties of reduced-fat Mozzarella cheese. Int Dairy J 14:161-172

Shinzawa H, Awa K, Kanematsu W, Ozaki Y (2009) Multivariate data analysis for Raman spectroscopic imaging. J Raman Spectrosc 40:1720-1725. doi:10.1002/jrs.2525

Upreti P, Metzger LE (2006) Influence of calcium and phosphorus, lactose, and salt-to-moisture ratio on cheddar cheese quality: manufacture and composition. J Dairy Sci 89:420-428. doi:10.3168/jds.S00220302(06)72106-3

Washam CJ, Kerr TJ, Hurst VJ, Rigsby WE (1985) A scanning electron microscopy study of crystalline structures on commercial cheese. Dev Ind Microbiol 26:749-761

Yiu SH (1985) A fluorescence microscopic study of cheese. Food Microstruct 4:99-106 